\title{
Transnationale Bündnisse von Gewerkschaften und NGOs. Strategien in Interaktion ${ }^{* *}$
}

\section{Zusammenfassung}

Globalisierte Produktionsmuster erschweren traditionelle Arbeiternehmervertretung und stellen Gewerkschaften und NGOs vor neue Herausforderungen. Private Governanceformen und die Nutzung von Konsumentenmacht stehen zur Debatte. Kooperationen von Gewerkschaften und NGOs gelten als erfolgsversprechende Bündnisse in einer globalen, unregulierten Ökonomie. Dieser Artikel analysiert die Strategien dieser neuen Bündnisse in der transnationalen Arbeitsregulierung. Strategien werden definiert als interaktive, multiple Sequenzen erfolgsorientierten Handelns im Zeitverlauf. Im Mittelpunkt dieses Beitrags stehen strategische Interaktionen, die zeigen, auf welche Weise Gewerkschaften und NGOs gemeinsam handeln - nicht trotz, sondern wegen originär unterschiedlicher strategischer Präferenzen. Anhand eines Fallbeispiels werden typische Strategien von Gewerkschaften und NGOs und ihr Zusammenspiel in der transnationalen Governancearchitektur von Arbeit nachgezeichnet.

Schlagwörter: Gewerkschaft, NGOs, Koalitionen, transnationale Regulierung von Arbeit

\section{Transnational Coalitions of Labour Unions and NGOs. Strategies in Interaction}

\section{Abstract}

Globalised production regimes hamper traditional ways of workers representation and present new challenges for labour unions and NGOs. Here, private forms of governance and consumer power are under discussion. Cooperation of labour unions and NGOs represent promising coalitions in a globalized, unregulated economy. This article analyses these new coalitions and their common strategies to transnationally regulate labour. Strategies are defined as interactive, multiple sequences of successoriented activities over time. This paper focuses specifically on strategic interactions that outline in which ways labour unions and NGOs act jointly - not despite, but because of originally different stra-

* Melanie Kryst, Universität Marburg, FB Gesellschaftswissenschaften und Philosophie, 35037 Marburg. E-Mail: melanie.kryst@gmx.de.

** Artikel eingegangen: 31.08.2017, revidierte Fassung akzeptiert nach doppelt-blindem Begutachtungsverfahren: 14.3 .2018 
tegic preferences. Utilizing a case study approach the article retraces typical trade union and NGO strategies and their interplay in the realm of transnational labour governance.

Key words: strategies, labour unions, NGOs, coalitions, interactive mechanisms, transnational labour governance (JEL: J 51; J 83)

\section{Einleitung}

In Zeiten der Globalisierung sehen sich Gewerkschaften und NGOs (Non-governmental organizations) weltweit verzweigten Produktionswegen gegenübergestellt, die traditionelle Arbeitnehmervertretung erschweren. Strategien von Arbeitsaktivisten in globalen Governancekontexten sind vielfältig. Der transnationale Raum wird von Gewerkschaften und NGOs erschlossen (Pries, 2010). Jenseits von Bemühungen um transnationalen sozialen Dialog oder (national)staatliche Regulierung entwickeln sich private Governanceformen, wie z.B. Labeling oder Verhaltenskodizes, und multinationale Unternehmen werden durch Konsumentenkampagnen unter Druck gesetzt. Der Markt wird zur neuen politischen Arena (Bartley, 2007; King \& Pearce, 2010).

Transnationale Kooperationen zwischen Gewerkschaften und NGOs werden als erfolgsversprechende Bündnisse in einer globalen, unregulierten Ökonomie gewertet (s. Übersicht in Fichter, Gester \& Zeuner, 2004). Sowohl in der Gewerkschafts- als auch in der Bewegungsforschung werden sie unter dem Vorzeichen einer möglichen Stärkung der Handlungsfähigkeit diskutiert. Implizit werden komplementäre Funktionen der beiden Akteursgruppen angenommen: Gewerkschaften als traditionelle Akteure der Arbeitsregulierung, stark verankert in den staatlichen Politikarenen und erprobt in Verhandlungen mit Unternehmen, scheinen ein ideales Pendant zu kleinen, schnell agierenden und medienerfahrenen NGOs zu bilden. Eine schlagkräftige Paarung kann entstehen. Bisherige Forschung und Praktikereinsichten beschäftigen sich vor allem mit der Entstehung und Gestalt von Gewerkschafts-NGOBündnissen (zum Beispiel Krüger, 2002; Anner \& Evans, 2004; Braun \& Gearhart, 2004; Egels-Zandén \& Hyllman, 2011). Es werden auf der einen Seite bündnisbildende Faktoren, wie zum Beispiel eine geteilte Zielperspektive (Gallin, 2000) oder Arbeitsweise (Della Porta, 2006), beschrieben. Auf der anderen Seite werden mögliche Konfliktpunkte betont, etwa durch eine unterschiedliche Organisationsidentität (Ford, 2009).

Wenig beachtet bleibt neben der Bündnisbildung das gemeinsame Handeln von Gewerkschaften und NGOs, das bisher nur in wenigen Ausnahmen untersucht wurde (Ford, 2009; Kryst, 2012; Egels-Zandén, Lindberg \& Hyllman, 2015). Dieser Beitrag geht der Frage nach, welche Strategien Gewerkschaften und NGOs gemeinsam in der transnationalen Governancearchitektur von Arbeit entwickeln. Mittels eines Ansatzes, der Strategien als Sequenzen erfolgsorientierten Handelns definiert (aufbauend auf Jasper 2004, 2012; Raschke \& Tils, 2013), werden Strategien in ihrem dynamischen, wechselseitigen Zusammenspiel erfasst. Eine induktive Bündnisanalyse zeigt, wie Gewerkschaften und NGOs in ihrer Strategiebildung den transnationalen sozialen beziehungsweise tripartistischen Dialog mit dem transnationalen Aktivismus kombinieren. Strategien von Gewerkschaften und NGOs befinden sich in Interaktion - nicht trotz, sondern wegen originär unterschiedlicher Präferenzen der Bündnispartner. 
Im Folgenden wird nach einer Gegenüberstellung der Ausgangsbedingungen für Gewerkschaften und NGOs und deren Zusammenspiel in Bündnissen ein interaktionistischer Strategiebegriff entwickelt, der auf jüngsten Forschungsansätzen aufbaut. Übersetzt in den Kontext der globalen Governancearchitektur von Arbeit ermöglicht ein Analyserahmen die Untersuchung von Gewerkschafts- und NGO-Strategien in Interaktionen. Aufbauend auf Ergebnissen einer exemplarischen Fallstudie des European Banana and Agro Industrial Product Action Networks (EUROBAN) (Kryst, 2017) werden zunächst Strategien des Gewerkschafts-NGO-Bündnisses typologisiert. In der Darstellung der Interaktionsmechanismen der beiden Strategietypen werden schließlich deren dynamischen Kombinationen und Interdependenzen benannt, und damit der interaktive Charakter der Strategien von Gewerkschaften und NGOs in transnationalen Bündnissen zur Arbeitsregulierung betont.

\section{Strategien von Gewerkschaften und NGOs in der transnationalen Governancearchitektur von Arbeit}

Sowohl die Gewerkschafts- als auch die Bewegungsforschung zeigt ein zunehmendes Interesse an Bündnisbildungen vor dem Hintergrund der Globalisierung. Die interagierenden Strategien der beiden Akteursgruppen - bislang noch mangels eines ausgeprägten Strategiebegriffs vernachlässigt - stehen im Fokus dieses Beitrags.

\subsection{Gewerkschafts-NGO-Bündnisse}

Bündnisse von Gewerkschaften und NGOs werden zumindest implizit unter der Annahme komplementärer Merkmale der ,alten' und ,neuen“ Akteure der Arbeitsregulierung beschrieben (z.B. in Krüger, 2002; Scherrer, 2002, S. 124-125). Gewerkschaften und NGOs sind in sich keine homogenen Gruppen, dennoch sind sie jeweils geprägt von charakteristischen Organisationsformen und Arbeitsweisen, Handlungssphären, sowie Problem- und Interessenlagen in der Arbeitsregulierung. Sie erscheinen damit als distinktive Bündnispartner, die die Problematik der globalen Arbeitsstandards auf unterschiedliche, aber potentiell kombinierbare Weise bearbeiten.

Gewerkschaften auf der einen Seite sind zentral durch ihre Mitglieder definiert, legitimiert und diesen gegenüber verpflichtet. Auf ihrer Primärmacht, den Beschäftigten in Unternehmen, gründet sich ihre Organisationsmacht, die als zentral für die Handlungsfähigkeit und Stärke von Gewerkschaften gegenüber Arbeitgebern gilt (Schroeder, 2014, S. 23). Als Interessengruppen bringen sie zudem die Interessen ihrer Mitglieder im politischen Willensbildungs- und Entscheidungsprozess zur Geltung. Die Verbesserung von Arbeitsbedingungen ist das Kerngeschäft von Gewerkschaften in den Betrieben, den Tarifverhandlungen oder dem politischen Raum - den drei klassischen Arenen gewerkschaftlichen Handelns (Schroeder, 2009). Als professionalisierte Organisationen verstanden, lassen sich Gewerkschaften durch eine definierte Anzahl an Mitgliedern, ein eher passives Mitgliederverständnis, gewählte Vertreter sowie Bürokratisierung und Zentralisierung charakterisieren (Avdagić \& Crouch, 2006).

Vor allem Gewerkschaften sind vor dem Hintergrund wirtschaftlicher Globalisierungsprozesse mit Problemen ihrer Einflussmöglichkeiten konfrontiert. Zum einen erodiert das 
Verhältnis zu Partnern in der politischen Sphäre (Evans 2010, S. 356), zum anderen sind Unternehmen durch globale Produktionsprozesse und die Logik des Wettbewerbsstaates weniger verpflichtungsfähig (Streeck, 1996, S. 186ff.). Gewerkschaften haben in dieser Situation sinkende Mitgliederzahlen zu verzeichnen (Streeck \& Rehder, 2005, S. 65). Internationales Gewerkschaftshandeln wird erschwert durch mangelhafte Organisationsstrukturen jenseits der nationalen Ebene (Koch-Baumgarten, 1999, S. 11f.) und verbleibt bisweilen als „Restposten“ (Reutter \& Rütters 2003, S. 512).

Dennoch, den Gewerkschaften kann noch immer eine Verhandlungsmacht in der globalen Governancearchitektur von Arbeit eingeräumt werden. Ansätze des pragmatischen Internationalismus messen Ergebnisse internationalen Gewerkschaftshandelns an zurückgeschraubten, realitätsnäheren Erwartungen hinsichtlich der Regulierungsdichte und -intensität. Gewerkschaften sind - so staatliche und gesellschaftliche Strukturen es ihnen ermöglichen - zentrale Akteure im Bereich der Selbstregulierung von Arbeit (Koch-Baumgarten, 2006, 2011). Sie tragen zu Komponenten einer transnationalen Governancearchitektur von Arbeit bei: durch Lobbyarbeit der globalen Gewerkschaftsverbände in internationalen Organisationen wie der Internationalen Arbeitsorganisation (ILO), durch internationale Rahmenvereinbarungen (IFAs) oder gar durch ein einzigartiges internationales Kollektivvertragssystem in der Transportschifffahrt sowie durch ihre Teilnahme an NGO-Bündnissen (Koch-Baumgarten \& Kryst, 2015). Diese besondere Rolle der Gewerkschaften sollte in Beschreibungen privater Governanceformen von Arbeit (Hassel, 2008) nicht weiter vernachlässigt werden.

NGOs auf der anderen Seite sind „Organisationen des ,dritten Sektors““(Janett, 1997), die sich zwischen die Sphären von staatlicher Gewalt und wirtschaftlicher Macht schieben. NGOs werden hier verstanden als der institutionelle Kern von sozialen Bewegungen (Cohen \& Rai, 2000, S. 12). Als Organisationen bilden sie das Gestell und den nach außen gerichteten Arm der sozialen Bewegung (Janett, 1997, S. 146), wobei professionelle Vereinigungen und Gewerkschaften von diesem Verständnis ausgeschlossen sind (Roth, 2005: 92ff.). Hinsichtlich der Ziele und Interessenlage lässt sich eine große Bandbreite von Themen ausmachen. Hier wird der Begriff eingeschränkt verwendet für politikorientierte NGOs (Heins, 2002, 132f.) in der Arbeitsregulierung, an anderer Stelle als „labour NGOs“ (Ford, 2009), oder „labor-based NGOs“ (Bronfenbrenner, 2007) beschrieben.

NGOs erfahren seit den 1990er Jahren verstärkte Aufmerksamkeit als relevante Akteure auf internationaler Ebene. Dahinter steht die Annahme, dass sie als zivilgesellschaftliche Akteure in der Weltpolitik zu mehr Demokratie und Effektivität in der Politikumsetzung verhelfen könnten (s. Übersicht in Klein, Walk \& Brunnengräber, 2005). Als NGONetzwerke mit spezifischen transnationalen Handlungsmöglichkeiten (Keck \& Sikkink, 1998) verorten sie sich in der transnationalen Politiksphäre. In der transnationalen Governancearchitektur von Arbeit bleiben sie bisweilen aber hinter den garantieren Einflusskanälen der Gewerkschaften, etwa bei der ILO, zurück (Koch-Baumgarten \& Kryst, 2015).

Besonders bei privaten Governanceformen werden NGOs als zentrale Akteure betont (Hassel, 2008): Die Unterstützung von Labels, die Forderung von Verhaltenskodizes, die Inszenierung von Protesten oder Kampagnen gegenüber transnationalen Unternehmen gehören zum Standardrepertoire von NGOs. Sie wirken mit diesen Instrumenten an der Institutionenbildung im Markt mit (Bartley, 2007; King \& Pearce, 2010). Durch die mangelhaf- 
te Umsetzung der freiwilligen Selbstregulierung von Unternehmen werden NGOs zudem erneut in regulatorischen Initiativen der Arbeitsregulierung in der staatlichen Sphäre aktiv (Kryst, 2012; Fransen \& Burgoon, 2015).

Bündnisbildung wird für beide Akteursgruppen als erfolgsversprechend eingestuft. Ein Bündnis wird hier definiert als eine abgrenzbare Organisationseinheit, die durch die Zusammenlegung von Ressourcen unabhängiger Organisationen aufgebaut wird (Mayer, 2009, S. 220). In der Gewerkschaftsforschung sind Zusammenschlüsse besonders auf globaler Ebene von Interesse (Müller, Platzer \& Rüb 2010), und „coalitional power“ (Brookes, 2013) wird als eine Machtbasis der Gewerkschaften beschrieben. In der Bewegungsforschung werden Bündnisse ebenfalls als relevant für den Erfolg der Bewegung angesehen, sind bislang aber erst in Ansätzen erforscht (Van Dyke \& McCommon 2010). Eine neue Perspektive auf Revitalisierungsstrategien der Gewerkschaften konstatiert Bündnisse mit NGOs als eine Möglichkeit der Gewerkschaften, sich neu zu behaupten (Baccaro, Haman \& Turner, 2003; Frege \& Kelly 2003). Parallel entsteht ursprünglich mit Blick auf Gewerkschaftshandeln im globalen Süden der Ansatz des Social Movement Unionism, der die Bedeutung der Arbeiterbewegung als politischen Bewegungsakteur in Erinnerung ruft und die Bündnisbildungen der Gewerkschaften mit sozialen Bewegungs- oder Gemeindeorganisationen betont (Bronfenbrenner, 2007; Fairbrother \& Webster, 2008).

Konkrete empirische Beispiele von Gewerkschafts-NGO-Bündnissen im Bereich der Arbeitspolitik finden sich verstärkt seit Beginn der 2000er Jahre (z.B. Krüger 2002; Anner \& Evans, 2004; Spooner, 2004; Arenas, Lozano \& Albreda, 2009). Die bisherigen Arbeiten und Praktikereinsichten sind erkenntnisreich, da sie Gemeinsamkeiten und Unterschiede der beiden Akteursgruppen herausarbeiten und daraus potentiell resultierende Spannungen sowie bündnisstärkende Faktoren betonen. Diese sind zum Beispiel funktionelle Faktoren auf Organisationsebene oder die Identität der Organisation (Braun \& Gearhart, 2004), die Verortung in den verschiedenen Sphären der Arbeitsregulierung (Egels-Zandén \& Hyllman, 2011, S. 254) oder die Zielsetzungen (Gallin, 2000, S. 28) und Interessenlagen von Gewerkschaften und NGOs (Compa, 2004). Nur wenige Ausnahmen erforschen bisher jedoch analytisch fundiert das gemeinsame Handeln von Gewerkschaften und NGOs anhand empirischer Fallbeispiele (Ford, 2009; Kryst, 2012; Egels-Zandén, Lindberg \& Hyllman, 2015).

Insgesamt zeigt sich in der bisherigen Forschung ein starker Fokus auf Entstehungsbedingungen von Bündnissen, wobei deren gemeinsames Handeln noch weitestgehend unerforscht bleibt. Es werden Gegensätze zwischen den beiden Akteursgruppen herausgearbeitet, und zugleich Möglichkeiten der Überwindung eben dieser aufgeführt. Unklar bleibt, wie sich das Handeln in Bündnissen kombinieren lässt - selbst, wenn die Akteursgruppen sich nicht auf eine gemeinsame Strategie einigen. Daher wird der Blick auf das Forschungsdesiderat des gemeinsamen strategischen Handelns von Gewerkschaften und NGOs in transnationalen Bündnissen der Arbeitsregulierung gerichtet, welchem im Folgenden ein interaktiver Strategiebegriff gerecht werden soll.

\subsection{Eine interaktionistische Strategieanalyse}

Lange Zeit war der Strategiebegriff in der politikwissenschaftlichen Forschung mit militärischem Handeln (Überblick: Münkler, 2010, S. 45) und den internationalen Beziehungen (Überblick: Schwarz, 2008, S. 102) verknüpft und fand später in der Spieltheorie Verwen- 
dung. Heute wird ein allzu negativ konnotiertes Strategieverständnis kritisiert (Saretzki, 2010, S. 125). Mit dem cognitive turn in den Sozialwissenschaften kommen schließlich Ansätze auf, die sich von bloßen interessengeleiteten Annahmen absetzen. An den Strategiebegriff knüpfen zunächst Ansätze der Politikfeldforschung an, die das Zusammenspiel von (kollektiven) politischen Akteuren untersuchen. Die Bedeutung von Werthaltungen, Argumenten und Ideen vor Kosten-Nutzen-Kalkulationen bei Entscheidungen in Politikfeldern werden betont (z.B. Sabatier, 1998).

Insbesondere in der deutschsprachigen politikwissenschaftlichen Forschung ist in den letzten Jahren ein Aufleben der Debatte über politische Strategie zu beobachten, wobei versucht wird, Grundlagen politischer Strategieanalysen (Raschke \& Tils, 2013; Wiesendahl, 2010) in die praxistaugliche Anwendung zu bringen. Diese beratungsorientierten Überlegungen fokussieren jedoch auf Regierungen und Parteien. Die Betonung kommunikativer Elemente führt zu Überschneidungen mit der Kommunikations- (Schmitt-Beck, 2008) und Political Leadership-Forschung (Glaab, 2008).

Für Gewerkschaften spielen Fragen der Strategie von Anbeginn der Arbeiterbewegung eine Rolle. Historisch ist die Strategie der Einheitsfront und der Volksfront in den 1920er und 1930er Jahren zu nennen (Abendroth 1969). Später wurde in der Gewerkschaftsforschung in den 1970er Jahren implizit die Strategiefrage im klassischen Gegensatz zwischen konfliktorischem und kooperativem Handeln gegenüber Arbeitgebern aufgegriffen (Bergmann, Jacobi \& Müller-Jentsch 1975, S. 26). Eine strenge bipolare Abgrenzung wird heute aber nicht mehr vertreten (Müller-Jentsch, 2008, S. 64ff.). Von strategic choice der Gewerkschaften wird jüngst vor allem vor dem Hintergrund schwindender Einflussmöglichkeiten der Gewerkschaften im globalen Raum und den daraus resultierenden Revitalisierungsstrategien gesprochen (Frege \& Kelly, 2003). Daneben werden vereinzelt Konzepte des Organisationslernens auf Gewerkschaften übertragen (Rehder, 2008).

In der Bewegungs- beziehungsweise NGO-Forschung sind Strategien implizit präsent bei der Frage der Aktionsformen, prominent als bewegungsspezifisches Aktionsrepertoire konzeptualisiert (Tilly, 1978). Daneben gehen Konzepte der politischen Gelegenheitsstrukturen (Eisinger, 1973), der Industrie- (King, 2008) und Unternehmensgelegenheiten (Schurman, 2004) zwar auf die Frage der günstigen Einflussmöglichkeiten von Bewegungsorganisationen ein, stellen selbst aber keine explizit strategischen Überlegungen an. Neuere Ansätze beginnen einen ausgeprägten Strategiebegriff zu entwickeln (Maney, Andrews, KutzFlamenbaum, Rohlinger \& Goodwin, 2012; Meyer \& Staggenborg, 2012), der die agency von Akteuren im Gegensatz zu deren strukturellen Beschränkungen betont und strategische Interaktionen ins Zentrum stellt (Jasper, 2004, 2012; Jasper \& Duyvendak, 2015).

Ich favorisiere einen Strategiebegriff, der sich von einem rein rationalen Verständnis abgrenzt. Letzteres begreift Strategien als rationales Wahlhandeln zur Erfolgsmaximierung in einer überschaubaren, kalkulierbaren Situation. Strategien seien demnach „,erfolgsorientierte Konstrukte, die auf situationsübergreifenden Ziel-Mittel-Umwelt-Kalkulationen beruhen“ (Raschke \& Tils, 2013, S. 127). Der erhoffte Erfolg in der Zukunft soll durch strategische Entscheidungen herbeigeführt werden (Wiesendahl, 2010, S. 21). Dieser Erfolgsorientierung wird hier als Definitionskriterium zwar zugestimmt. Doch ein enges Strategieverständnis eignet sich vor allem dazu, ein instrumentelles Kalkül im Handeln von politischen Akteuren auszumachen (Raschke \& Tils, 2013, 88) und dieses in der politischen Praxis als 
Handreichung für Politiker vorzuschlagen. Für eine empirische Strategieanalyse ist der Begriff aber zu eingeschränkt.

Strategisches Handeln wird hier als soziales und interaktives Handeln definiert. Bei dem Versuch, ihre Ziele in verschiedenen Arenen durchzusetzen, stellen sich strategisch handelnde Akteure auf ihre Adressaten, Gegenspieler, andere institutionenbildende Akteure oder Bündnispartner ein (Jasper, 2004, S. 6). An ihnen wird strategisches Handeln ausgerichtet. Auf Grundlage der bisherigen Literatur definiere ich Strategien daher als interaktive, multiple Sequenzen erfolgsorientierten Handelns im Zeitverlauf. Aus dem hier verwendeten Verständnis leite ich analytisch einen Dreiklang von Strategieelementen ab. Strategien enthalten Bemühungen, um andere zum gewünschten Handeln zu bewegen: „By strategic action, I mean simply a situation in which individuals or groups try to get others to do what they want them to" (Jasper, 2012, S. 24f). Das heißt, es lassen sich analytisch a) ausgewählte Aktionen bzw. Instrumente der strategisch handelnden Akteure benennen, die b) an einen bestimmten Adressaten gerichtet und c) mit inhaltlichen Forderungen ausgestattet sind (s. Abb. 1).

Die Bezugnahme auf einen interaktiven und dynamischen Strategiebegriff ermöglicht Forschung zu Entwicklungen im Zeitverlauf unter Berücksichtigung sich verändernder Akteurskonstellationen. Strategien konkretisieren sich in ,sequences of events“ (Maney et al., 2012, S. 171), die zur Zielerreichung beitragen. Sie sind hier nicht als stringent planbarer Ablauf konzipiert, sondern werden als Prozess verstanden und können parallele und sogar widersprüchliche Sequenzen enthalten. Das heißt weiter, sich in Aktionen, Adressaten und Forderungen manifestierende Strategien stehen in ständiger Interaktion miteinander und bedingen oder behindern sich gegenseitig - ein Zusammenhang, der insbesondere bei gemeinsamen Strategien mehrerer Akteure auftritt. Diese Interaktionsmechanismen sind daher von zentraler Bedeutung, wenn es um die Frage von dynamischer Strategieentwicklung zwischen den Bündnispartnern geht. Mechanismen sind wiederkehrende Prozesse, die spezifische Bedingungen und Ergebnisse verbinden und zeigen, wie diese durch Zwischenschritte zustande kommen (Mayntz, 2004, S. 241). Es gilt nun in der folgenden empirischen Analyse eines Gewerkschafts-NGO-Bündnisses, die Strategien und ihre Interaktionsmechanismen im Zeitverlauf zu identifizieren (s. Abbildung 1).

Abbildung 1: Analyserahmen: Interaktionistischer Strategieansatz

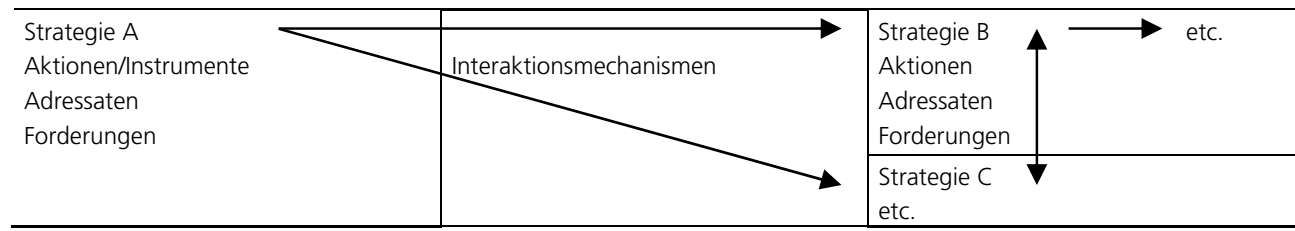

Quelle: Eigene Darstellung.

Im Folgenden werden, einer induktiven Analyse folgend, Strategien von Gewerkschaften und NGOs anhand dieses Ansatzes typologisiert und Interaktionsmechanismen benannt. Als empirische Grundlage dient dabei eine qualitative Fallstudie, die einen exemplarischen Fall mit möglichst großem Erkenntniswert untersucht. Dafür bietet sich ein besonders langfristiges Gewerkschafts-NGO-Bündnis an. Das European Banana Action Network (EURO- 
BAN), gegründet 1994 (Kryst, 2017), wird als europäisch basiertes Bündnis ausgewählt, das sich mit einer transnationalen Ausrichtung um die (Durch-)Setzung von Arbeitsstandards bemüht. EUROBAN ist ein Bündnis von Gewerkschaften und NGOs in Europa, zentrale Organisationen sind beispielsweise Banana Link, Fair Trade International oder Peuples Solidaires. Wichtige Partner sind der IUF (International Union of Food, Agricultural, Hotel, Restaurant, Catering, Tobacco and Allied Workers' Association), daneben Partnerorganisationen in den USA und Lateinamerika. EUROBAN arbeitet mit dem Fokus auf den globalen Bananenhandel und steht einer globalisierten und weitgehend unregulierten Produktionskette gegenüber.

Ziel der folgenden Fallstudie ist es, durch eine ,within-case“-Analyse (George \& Bennett, 2005, S. 19) typische Strategien zu identifizieren und Interaktionsmechanismen durch Process Tracing nachzuzeichnen (George \& Bennett, 2005, S. 12f.). Als Datengrundlage dient eine Dokumentenanalyse von öffentlichen Dokumenten, wie zum Beispiel Newslettern, Pressemitteilungen, Kampagnenmaterial oder Webseiten, sowie von internen Dokumenten, wie zum Beispiel Protokollen, Präsentationen, internen Konzeptpapieren und EMails. Ergänzend wurden acht leifadenstrukturierte Experteninterviews mit Mitgliedern des Bündnisses zwischen Oktober 2013 und Mai 2014 durchgeführt. Eine inhaltsanalytische Auswertung ermöglichte die induktive Bildung von Strategietypen, Rückschlüsse auf Voraussetzungen der einzelnen Strategieelemente, sowie die Analyse der Interaktionsmechanismen der Strategien im Zeitverlauf.

\section{Ergebnis: Strategien des Dialogs und des Aktivismus in Interaktion}

Welche Strategien entwickeln Gewerkschaften und NGOs gemeinsam in der transnationalen Governancearchitektur von Arbeit? Das folgende Kapitel widmet sich der Typologisierung unterschiedlicher strategischer Aktionen, Adressaten und Forderungen von Gewerkschaften und NGOs in der transnationalen Governancearchitektur von Arbeit, um darauffolgend Mechanismen der Strategieinteraktion in Gewerkschafts-NGO-Bündnissen zu identifizieren.

\subsection{Gewerkschafts- und NGO-Strategien: Gegensätze und Graubereiche}

Die Analyse erfolgt im Kräftefeld der Erwerbsregulierung, in dem die Aushandlung, Festlegung und Kontrolle von Arbeitsbedingungen der Erwerbstätigen stattfindet (Pries, 2008, S. 298). Innerhalb dieses Rahmens beschränke ich mich auf das öffentliche Regime, welches Handlungsnormen für eine generelle Norm- und Regelorientierung für Erwerbsarbeit beinhaltet (Pries, 2010, S. 31). Für die transnationale Erwerbsregulierung liegt eine lückenhafte Governancearchitektur vor (Koch-Baumgarten 2006, 2011). Im Gegensatz zu Regulierungen durch eine Zentralgewalt lenkt die Governanceperspektive sinnvollerweise das Augenmerk auf den „Flickenteppich“ (Koch-Baumgarten, 2006, S. 211) der minimalistischen und informellen Regulierungen, bis hin zu ,kleinen“ Koordinationsansätzen (Koch-Baumgarten, 2006, 219). In diesem politisch-institutionellen Aushandlungsprozess der Normsetzung (Bartley, 2007) positionieren sich widerstreitende Akteure mit ihren Strategien. 
Hinsichtlich der gewählten Strategien dominierten bei EUROBAN neben kontinuierlicher Kampagnenarbeit von Beginn an Lobbybemühungen. Anfang der 1990er Jahre brachte sich EUROBAN in die Debatte um ein neues Importregime für Bananen in der EU ein und forderte 1994 den bevorzugten Zugang für sozial-ökologisch produzierte Bananen (Smith, 2015, S. 63). Das Bündnis unterstützte durch Postkartenkampagnen und Lobbying das zu dieser Zeit entstehende Fairtrade-Label für Bananen zulasten alternativer Ansätze der Weltläden, wie sie von Gebana oder Oxfam bereits bestanden (Interview 10.02.2014, Paris). Insbesondere von Gewerkschaftsseite wird diese marktbasierte Lösung dauerhaft intern kritisiert, da sie nicht auf der zwingenden Einbeziehung von Gewerkschaftsrechten aufbaut, wenngleich Fairtrade nach außen von EUROBAN weiter als potentielle Lösung propagiert wird (Interview 25.03.2014, Genf; Interview 14.05.2014, Prag).

Erste Unternehmenskampagnen von EUROBAN adressierten 1997 Del Monte und 1998 Chiquita. Parallel zur Druckausübung auf multinationale Unternehmen wurden diese in Dialogformate eingebunden: 1998 richtete EUROBAN eine erste internationale Bananenkonferenz aus, 2000 ein Treffen mit den vier größten Bananenproduzenten. Folgende Verhandlungen führten 2001 schließlich zur Unterzeichnung der Internationalen Rahmenvereinbarung zwischen dem IUF und Chiquita (Riisgaard, 2004). Ende der 1990er Jahre wurden von EUROBAN erstmals Supermärkte als neue Adressaten aufgegriffen im Rahmen einer Kampagne gegen Tesco und Asda (Smith 2015, S. 89 et seq.), welche in der Folge Mitglieder der neu gegründeten Ethical Trading Initiative wurden. 2002 übte EUROBAN Druck auf das Unternehmen Noboa aus, jedoch ohne Erfolg (Frundt, 2009, S. 190). Erneuter Druck von außen trifft 2006 das Unternehmen Dole (Interview 10.02.2014, Paris). Die jüngste Make Fruit Fair-Kampagne von NGO-Bündnismitgliedern von EUROBAN adressiert große Supermärkte als verantwortliche Akteure für die Durchsetzung von Arbeitsstandards entlang der globalen Lieferkette (Interview 14.04.2014, Paris; Interview 25.03.2014, Genf).

Insbesondere Partnergewerkschaften von EUROBAN sprachen sich daneben für den Ausbau der Dialogstrategie des Bündnisses aus (Interview 25.03.2014, Genf; Interview 10.02.2014, Paris). 2005 wurde die zweite internationale Bananenkonferenz durchgeführt, auf der EUROBAN deutlich härtere Forderungen als auf der ersten stellte (Smith, 2015, S. 75). Das Bündnis brachte einen konkreten Gegenentwurf für ein Handelsregime in die Debatte ein (Parker \& Harrison, 2004) und schlug ein internationales Bananenabkommen vor, das Unternehmen auf die ILO-Kernarbeitsnormen verpflichtet (Smith, 2015, S. 121 et seqq.). EUROBAN war in der Folge maßgeblich am Aufbau des seit 2009 bestehenden World Banana Forums beteiligt, einem Dialogforum (Interview 23.10.2013 (b), Rom), das sich zum andauernden Austausch in Arbeitsgruppen von Gewerkschaften, NGOs und Unternehmen gebildet hat (Interview 23.10.2013 (b), Rom). EUROBAN versuchte zudem staatliche Akteure zu integrieren, allerdings mit nur sporadischem Erfolg (Interview 23.10.2013 (a), Rom). Das World Banana Forum fand danach 2012 und zuletzt 2017 statt.

Für eine Strategieanalyse des EUROBAN-Bündnisses werden hier als erstes Strategieelement Aktionen (Meyer \& Staggenborg, 2012, S. 8f.) beziehungsweise Instrumente (Zimmer \& Speth, 2009, S. 287) benannt. In diesen Mitteln oder Vorgehensweisen (Wiesendahl, 2010, S. 27) manifestiert sich die Art und Weise der Strategie. Im Fall von EUROBAN kann induktiv in Insider- und Outsider-Aktionen des Bündnisses unterschieden 
werden (s. Abb. 2). Als Insider-Aktionen werden dabei Aktionen kodiert, die auf Verhandlungen und beidseitige Abkommen abzielen. Empirische Beispiele von EUROBAN sind etwa Gespräche, Konferenzen oder Pilotprojekte, daneben die Beteiligung an Zertifizierung und Monitoring in Multistakeholder-Initiativen, die Verhandlungen für internationale Rahmenvereinbarungen, sowie Lobbying auf nationaler und internationaler Ebene. OutsiderAktionen hingegen sind solche Aktionen, die auf Veränderungen durch einseitige Zugeständnisse über öffentlichen Druck beziehungsweise über alternative Marktformen abzielen. Beispiele aus dem Fallbeispiel sind Kampagnen und Proteste, sowie die Unterstützung von Marktalternativen durch Buycott, dem gegenteiligen Boycott, oder Informationen über Labels wie Fairtrade. Streiks, als klassisches Gewerkschaftsinstrument, können als eine spezifische Outsider-Aktion beschrieben werden, die stets mit Verhandlungen in Zusammenhang stehen. Während Insider-Aktionen eher - aber nicht nur - durch Gewerkschaften getragen werden, die über das notwendige Netzwerk, die Machtbasis und das Selbstverständnis als Verhandlungspartner verfügen, sind Outsider-Aktionsformen das angestammte Repertoire von NGOs, die auf Skandalisierung und einer Rolle als watch dog aufbauen.

In der Theoretisierung der Ergebnisse bildet sich eine grundlegende strategische Frage gegenüber den Adressaten ab: Miteinander oder gegeneinander? (Raschke \& Tils, 2013, S. 374). Sowohl in der Gewerkschafts- als auch in der NGO-Forschung schlägt sich diese strategische Entscheidung implizit nieder. Dies ist etwa der Fall, wenn zwischen konfliktorischen und kooperativen Gewerkschaften (Bergmann, Jacobi \& Müller-Jentsch, 1975) unterschieden wird. Ebenso wird beim Ansatz des Social Movement Unionism nach strategischen Aktionen gesucht, die die Bewegungscharakteristiken von Gewerkschaften stärken (Fairbrother \& Webster, 2008, S. 211). Daneben unterscheidet die Bewegungsforschung diverse Aktionsformen und sucht nach der Ausbildung von verbandlichen Merkmalen. Es findet sich eine häufig getroffene Unterscheidung in direkte Taktiken und Lobbyismus respektive Routinepolitik (z.B. Clemens, 2005, S. 354). Die empirisch auftretenden OutsiderAktionen weisen auf die Relevanz markt-basierter Aktionen hin. Bei diesen üben Aktivisten öffentlichen Druck auf Unternehmen aus und werden bisweilen als Entrepreneurs aktiv und nehmen eine innovative Rolle im Institutionenaufbau im Markt selbst ein (Bartley, 2007, S. 310). Die Etablierung eines Nischenmarktes durch so genannte Marktbewegungen, durch Labelling- und Zertifizierungsinitiativen, bringen Druck auf Unternehmen durch den Marktmechanismus selbst (King \& Pearce, 2010).

Als weiteres Strategieelement konzeptualisiere ich Adressaten. Adressaten sind die jeweiligen Akteure, an welche sich eine Strategie richtet. Sie werden in bestimmten Arenen (Meyer \& Staggenborg, 2010, S. 8) ausgewählt (s. Tabelle 1). Bei EUROBAN werden zum einen Adressaten innerhalb der Arena der industriellen Beziehungen beziehungsweise des Tripartismus gewählt. Empirische Beispiele sind internationale Organisationen, wie die EU oder die ILO, daneben Regierungen oder Politiker im globalen Norden oder Unternehmensverbände. Auf der anderen Seite richtet EUROBAN sich über die Öffentlichkeit an Adressaten, die unter Druck gesetzt werden sollen. Adressaten in der öffentlichen Marktarena sind zum Beispiel multinationale Unternehmen, Fabriken oder Konsumenten. Daneben sind indirekt vor allem nationale staatliche Akteure mit öffentlichem Druck konfrontiert. Bei ersteren Adressierungen zeigen sich vor allem Gewerkschaften als relevante Akteure, die auf industriellen Beziehungen und einer sozialen Dialogkultur aufbauen können. Bei 
letzterer Adressierung sind es vor allem NGOs, die sich an politischen Gelegenheiten und Marktgelegenheiten orientieren.

Dieses Ergebnis bedeutet den Markt als neue Governancearena zu verstehen. Sowohl in der Verbände- als auch in der Bewegungsforschung wird der Staat häufig als primärer Adressat des Handels beschrieben, etwa im Tripartismus, durch Lobbying auf internationaler Ebene (Zimmer \& Speth, 2009), sowie beim Ansatz der politischen Gelegenheitsstruktur (Eisinger, 1973). Gewerkschaften tragen aber per se als Akteure der Selbstregulierung in der Erwerbsarbeit gemeinsam mit Unternehmen zu privaten Governanceformen bei (KochBaumgarten, 2011). Viele Forschende gehen im Zuge des Governancewandels zumindest implizit von einer staatlichen Regulierungsschwäche und einer Hinwendung zu Unternehmen aus. So machen soziale Bewegungsforscher ihre Theorien anschlussfähig für Analysen, die sich über die Arena der staatlichen Politik hinaus dem Markt zuwenden. Forschung zu Industrie- (King, 2008) oder Unternehmensgelegenheiten (Schurman, 2004) betont den Markt als neue politische Arena. Neuere Ergebnisse zeigen zudem überlappende Formen von staatlicher und nicht-staatlicher Normsetzung durch Arbeitsaktivisten auf (Fransen \& Burgoon, 2015; Kryst \& Zajak, 2017).

Schließlich beschreiben die Forderungen die inhaltliche Dimension der Strategie (Raschke \& Tils, 2013, S. 257). Als spezifische Forderungen bei EUROBAN erkenne ich konkrete, meist moderate Forderungen, die sich auf einen begrenzten Aspekt oder Raum zur Verbesserung von Arbeitsstandards in naher Zukunft beziehen (s. Tabelle 1). Beispiele sind etwa konkrete Lohnforderungen und spezifische Gewerkschaftsrechte, wie zum Beispiel Vereinigungsfreiheit, Versammlungsfreiheit und das Recht auf kollektive Verhandlungen oder andere konkrete Aspekte von Arbeitsstandards, die oftmals auf einer der ILOKernarbeitsnormen fußen. Im Gegensatz dazu kodiere ich umfassende Forderungen, welche allgemeine und weitreichende Forderungen sind, die sich auf einen grundlegenden Wandel im globalen Produktionsprozess beziehen. Diese beiden müssen sich nicht ausschließen, im Gegenteil: Spezifische Forderungen können als Etappen-, umfassende Forderungen als Langfristziele gelten. Beispiele für Letztere sind Forderungen, die auf die allgemeine Verbesserung von Arbeitsstandards hinweisen oder weitere Themen wie Umweltschutz aufnehmen. Im Resultat werden konkrete Forderungen vor allem von Gewerkschaften vertreten, die einer bottom up-Ideologie folgen und sich an Arbeitnehmerinteressen orientieren, während umfassende Forderungen durch NGOs getragen werden, die einem regulativen Ansatz der Arbeitsregulierung folgen und eher Idealen verpflichtet sind.

Grundsätzlich besteht damit hinsichtlich der Forderungen eine Unterscheidung von nicht notwendigerweise gegensätzlichen - spezifischen Zielen und weitreichenden Forderungen der strategischen Akteure (Maney et al., 2012, S. 17; Raschke \& Tils, 2013, S. 257). Dies bedeutet ein Kontinuum von Gewerkschafts- und NGO-Forschung: Für die Gewerkschaften sind meist die spezifischen Interessen von ArbeitnehmerInnen (Ford, 2009, S. 9), oft orientiert an den ILO-Kernarbeitsnormen (Ehmke, Simon \& Simon, 2009, S. 14) eine Ausgangsbasis, die über weiterreichenden Zielvorstellungen dominieren. Bei Untersuchungen zu Labour-NGOs werden neben den spezifischen Interessen ebenso radikalere oder weitreichendere Forderungen (z.B. Spooner, 2004, S. 22ff.) genannt. 
Tabelle 1: Strategien der Arbeitsregulierung in Gewerkschafts-NGO-Bündnissen

\begin{tabular}{|l|l|l|}
\hline $\begin{array}{l}\text { Strategie- } \\
\text { elemente }\end{array}$ & \multicolumn{1}{|c|}{$\begin{array}{c}\text { Strategien des transnationalen sozialen } \\
\text { bzw. tripartistischen Dialogs }\end{array}$} & Strategien des transnationalen Aktivismus \\
\hline Aktionen & In Bündnissen eher verfolgt von Gewerkschaften & \multicolumn{1}{|l|}{ In Bündnissen eher verfolgt von NGOs } \\
\hline Adressaten & $\begin{array}{l}\text { Insider-Aktionen } \\
\text { triellen Beziehungen bzw. der staatlichen Arena }\end{array}$ & $\begin{array}{l}\text { Outsider-Aktionen } \\
\text { der Marktarena }\end{array}$ \\
\hline Forderungen & Spezifische Forderungen & Umfassende Forderungen \\
\hline
\end{tabular}

Quelle: Eigene Darstellung.

Aktionen, Adressaten und Forderungen stehen in einem Zusammenhang zueinander. Im Ergebnis zeigt sich, dass auf der einen Seite Insider-Aktionen eher mit spezifischen Forderungen einhergehen und sich vor allem an Adressaten innerhalb der industriellen Beziehungen, wenn möglich auf transnationaler Ebene, beziehen. Diese Strategieelemente fügen sich zu einer hier benannten Strategie des transnationalen sozialen beziehungsweise tripartistischen Dialogs zusammen. Auf der anderen Seite werden Outsider-Aktionen eher an die Öffentlichkeit oder an Adressaten innerhalb der globalen Marktarena gerichtet und enthalten umfassendere Forderungen. Diese Zusammenhänge werden unter der hier benannten Strategie des transnationalen Aktivismus zusammengefasst. Es zeigt sich bei den Strategien von EUROBAN eine Tendenz von Gewerkschaften innerhalb der Bündnisse Strategien des transnationalen sozialen oder tripartistischen Dialogs zu verfolgen, während NGOs eher Strategien des transnationalen Aktivismus bevorzugen. Insgesamt gibt es jedoch Überschneidungen, sodass nicht immer eine eindeutige Zuordnung vorgenommen werden kann (s. Tabelle 1).

\subsection{Interaktionsmechanismen der Strategien in Gewerkschafts-NGO- Bündnissen}

Die beiden genannten Typen bilden das Kontinuum an variierenden Strategien von Gewerkschafts-NGO-Bündnissen in der transnationalen Governancearchitektur von Arbeit ab. Eine Analyse der interagierenden Strategien von Gewerkschaften und NGOs ermöglicht es, spezifische Abfolgen und Kombinationen zu identifizieren und damit Interaktionsmechanismen zu erläutern. Der Fokus dieser Analyse hebt sich damit ab von der Konzentration auf gegensätzliche oder gemeinsame Strategien von Gewerkschaften und NGOs und daraus potentiell resultierende Konflikte oder Erfolge. Reaktionen der Gegenseite, die zurück in die strategischen Entscheidungen der Akteure einfließen (Balsiger, 2015; Jasper \& Duyvendijk, 2015), sind hier nicht Gegenstand der Analyse. Ich differenziere als Ergebnis des Process Tracings bei EUROBAN induktiv insgesamt fünf Interaktionsmechanismen zwischen den beiden Strategietypen (Kryst, 2017), wovon hier beispielhaft zwei ausgeführt werden:

a) Verhandlungen durch Kampagnen: Im Laufe der Kampagnentätigkeiten von EUROBAN nutzte das Bündnis öffentlichen Druck gegen große Bananenproduzenten, wie Chiquita, Del Monte, Noboa oder Dole, um - im Sinne eines Bumerangmodells (Den Hond \& de Bakker, 2012) - zu Verbesserungen der Arbeitsbedingungen bei deren Zulieferern und Plantagen vor Ort zu gelangen. Dabei interagierten Strategien des transnationalen Aktivis- 
mus mit Strategien der transnationalen industriellen Beziehungen: EUROBAN versuchte, durch Druck auf Unternehmen von außen zu Verhandlungen und schließlich zu bindenden Abkommen zu gelangen (Tabelle 2).

Nach einer ersten Phase des Lobbyings, richteten sich Mitglieder von EUROBAN Ende der 1990er Jahre mittels koordinierter öffentlichkeitswirksamer Kampagnen an verschiedene multinationale Unternehmen in der Bananenproduktion. 1998 startete EUROBAN eine großangelegte Kampagne gegen Chiquita mit Aktivitäten in Lateinamerika und Europa. Das zu der Zeit angegriffene Markenimage von Chiquita bot sich für EUROBAN als geeignete Gelegenheit dar (Smith 2015, S. 82). EUROBAN unterstützte damit die Gewerkschaften in ihrer Verhandlungsmacht: ,the campaign would not stop so long as the company refuses to sit down with the Latin American unions“ (Smith, 2015, S. 85). Nach mehreren Verhandlungen mit dem Unternehmen führte der konstante Druck schließlich 2001 zur Unterzeichnung des ersten IFAs in der Agrarbranche durch Chiquita und den IUF (Riisgaard, 2004). Die Kampagnenarbeit und die Mobilisierung von Konsumenten war ein entscheidender Treiber für die erfolgreichen Verhandlungen (Interview 10.02.2014, Paris). Die Gewerkschaften hingegen konnten die notwendige Verhandlungslegitimität aufbringen und boten ein hilfreiches Netzwerk für die Umsetzung des Abkommens auf lokaler Ebene: „We knew [...] that we had affiliates in most of the countries where Chiquita was owning and growing bananas, that we would be able to work to actually ensure the implementation of the agreement" (Interview 25.03.2014, Genf). Hier wird deutlich, wie NGOs mit Druck von außen dazu beitragen, eine gewerkschaftliche Strategie für transnationale Kollektivverhandlungen erfolgreich umzusetzen.

Darüber hinaus adressierte das Bündnis 2006 das Unternehmen Dole. Das bestehende IFA mit Chiquita schien in dieser Zeit innerhalb des Unternehmens in Frage gestellt, und EUROBAN wollte unter anderem dieses Ergebnis nicht durch einseitigen Kampagnendruck auf einen der beiden Wettbewerber gefährden: „It is also clear that, as [...] the Agreement with COLSIBA and IUF is called into question internally within the company [Chiquita, Anm. d. Aut.] - that pressure on Chiquita's main competitor would be tactically helpful [...] It is also the most ,reactionary' of the big companies" (EUROBAN, 2003, S. 88). Dies führte zu einer Diversifikationsstrategie und einer öffentlichkeitswirksamen Kampagne gegen den Hauptkonkurrenten Dole. Ein Wechsel von Outsider- und Insider-Aktionen gegenüber Dole fand auch in diesem Fall mehrfach zeitlich eng aufeinander und kausal interagierend statt. Der Druck wurde damit industrieweit auf multiplen Angriffspunkten ausgeweitet, um zu Insider-Verhandlungsstrukturen mit Unternehmen über Arbeitsbedingungen zu gelangen.

Zusammengefasst machte der konstante Druck den Dialog mit Unternehmen erst möglich. Diese Abbildung von ,sequential patterning of tactics“ (Den Hond, de Bakker \& de Haan, 2010, S. 648) zeigt eine wiederkehrende Reihenfolge von Aktionsformen. Darüber hinaus wurde Kampagnendruck auf Wettbewerber ausgeweitet und spricht für einen industrieweiten Ansatz bei der Betrachtung von Strategien von Arbeitsaktivisten (Den Hond, de Bakker, \& de Haan, 2010). In den hier genannten Beispielen wirkten Kampagnen stets auf Verhandlungsbemühungen ein. Dieser Mechanismus, der sich teilweise über mehrere Kampagnen- und Verhandlungsrunden entfaltete, bestärkt einen dynamischen Ansatz, um diese Strategien von NGOs und Gewerkschaften in Interaktion zu erklären. 
Tabelle 2: Interaktionsmechanismus a) Verhandlungen durch Kampagnen

\begin{tabular}{|c|c|c|}
\hline Jahr & $\begin{array}{l}\text { Strategien für transnationalen sozialen } \\
\text { bzw. tripartistischen Dialog }\end{array}$ & $\rightarrow$ Strategien für transnationalen Aktivismus \\
\hline 1998 & & $\begin{array}{l}\text { Aktionen: NGO-unterstütze Kampagne gegen } \\
\text { Chiquita; } \\
\text { Adressierung: Chiquita wird auf transnationaler } \\
\text { Ebene adressiert } \\
\text { Forderungen: EUROBAN fordert Dialog des Unter- } \\
\text { nehmens mit Gewerkschaften über Arbeitsstan- } \\
\text { dards }\end{array}$ \\
\hline 2001 & $\begin{array}{l}\text { Aktionen: Verhandlungen mit bananenproduzie- } \\
\text { renden Unternehmen und Abschluss einer interna- } \\
\text { tionalen Rahmenvereinbarung mit Chiquita } \\
\text { Adressierung: IUF und Chiquita als Verhandlungs- } \\
\text { partner } \\
\text { Forderungen: spezifische Forderungen von einzel- } \\
\text { nen Arbeitsstandards sind Gegenstand der Ver- } \\
\text { handlungen }\end{array}$ & $\begin{array}{l}\text { Interaktionsmechanismus: Kampagnendruck bringt } \\
\text { Unternehmen an den Verhandlungstisch }\end{array}$ \\
\hline 2006 & $\begin{array}{l}\text { Interaktionsmechanismus: Kampagnendruck wird } \\
\text { auf einen zusätzlichen Adressaten der Industrie } \\
\text { ausgeweitet, um vorheriges Abkommen zu sichern }\end{array}$ & $\begin{array}{l}\text { Aktionen: Kampagnen gegen Dole, den } \\
\text { Hauptwettbewerber von Chiquita } \\
\text { Adressierung: Dole wird auf transnationaler Ebene } \\
\text { adressiert } \\
\text { Forderungen: allgemeine Verbesserungen der Ar- } \\
\text { beitsstandards }\end{array}$ \\
\hline
\end{tabular}

Quelle: Eigene Darstellung.

b) Verhandlungen statt Kampagnen: In umgekehrter Weise wurden bei EUROBAN im Laufe des Bündnisbestehens Verhandlungsergebnisse mit Adressaten geschützt, indem konfrontative Aktionen gegenüber diesen eingestellt wurden. Etablierte Dialoge mit Unternehmen waren bei EUROBAN nicht automatisch gefeit vor öffentlichkeitswirksamen Aktionen - im Gegenteil, der Druckaufbau war, wie gezeigt, teils inhärenter Teil der Verhandlungsstrategie. Dennoch gab es in jüngster Zeit einen anders gelagerten Interaktionsmechanismus, bei dem Strategien des transnationalen sozialen Dialogs auf Strategien des transnationalen Aktivismus des Bündnisses wirken: EUROBAN schützte den etablierten Dialog mit bananenproduzierenden Unternehmen im Rahmen des World Banana Forums und griff stattdessen Supermärkte mit der konfrontativen Make Fruit Fair-Kampagne an (Tabelle 3).

Bereits frühzeitig versuchte EUROBAN in einen langfristigen Dialog mit Produzenten $\mathrm{zu}$ treten und erreichte die Umsetzung von internationalen Bananenkonferenzen und die Etablierung des World Banana Forums im Jahr 2009. Insbesondere für Gewerkschaften und Produzenten aus dem globalen Süden zeigte sich eine Präferenz für diese Strategie des Dialogs (Interview 12.02.2014, Norwich). Es setzte sich im Bündnis die Idee durch, gemeinsam mit Unternehmen zu arbeiten: ,You change your main operation mode from one of automatic citicism to: 'Ok, let us work on the problems together.' So you become a partner in the solution, not just in raising awareness of the problem“ (Interview, 14.02.2014, Paris). Um den etablierten Dialog nicht durch öffentliche Kampagnen zu gefährden, entschied sich EUROBAN Outsider-Aktionen nicht mehr länger gegen solche Unternehmen anzuwenden, mit denen sich das Bündnis im Dialog befand. Das Risiko des Vertrauensverlustes wurde 
als zu hoch eingeschätzt: „You really think twice because you do not want to break the dialogue that you set up. So you basically do not want to really target the ones you speak to and you start to develop some relationship“ (Interview 14.05.2014, Prag).

Gleichzeitig wurden Outsider-Aktionen jedoch nicht aufgegeben; sie gehören zu den Kernaktionen von einigen Mitglieds-NGOs: ,, a lot of NGOS are at their heart campaigning organisations“ (Interview 12.03.2014, Norwich). Dies führte zu einer komplementären Neuausrichtung gegenüber neuen Adressaten. In der Folge wurden daher Großhändler beziehungsweise Supermärkte als neue Adressaten für Kampagnen des Bündnisses gewählt. Einige NGOs der Koalition starteten die Make Fruit Fair-Kampagne, die Supermärkte angreift und gleichermaßen staatliche Akteure auffordert, deren Supermarktmacht zu regulieren. Somit führten Verhandlungen zunächst zu einer Vermeidung von Kampagnen gegenüber den in Dialoge eingebundenen Unternehmen, und schlussendlich zu einem Adressatenwandel des Bündnisses.

Tabelle 3: Interaktionsmechanismus b) Verhandlungen statt Kampagnen

\begin{tabular}{|l|l|l|}
\hline Jahr & \multicolumn{1}{|c|}{$\begin{array}{c}\text { Strategien für transnationalen sozialen } \\
\text { bzw. tripartistischen Dialog }\end{array}$} & Strategien für transnationalen Aktivismus \\
\hline 2009 & $\begin{array}{l}\text { Aktionen: Dialog im World Banana Forum; } \\
\text { Adressierung: multinationale Unternehmen werden } \\
\text { als Verhandlungspartner zur Zusammenarbeit } \\
\text { adressiert } \\
\text { Forderungen: zunächst moderate Forderungen, um } \\
\text { Dialog nicht zu gefährden }\end{array}$ & \\
\hline 2011 & $\begin{array}{l}\text { Interaktionsmechanismus: Dialog wird geschützt, } \\
\text { keine Kampagnen gegen am World Banana Forum } \\
\text { beteiligte Unternehmen }\end{array}$ & $\begin{array}{l}\text { Aktionen: Kampagnen gegen Unternehmen wer- } \\
\text { den eingestellt; Kampagnen bleiben als Aktions- } \\
\text { form bestehen } \\
\text { Adressierung: Supermärkte als neue Adressaten } \\
\text { Forderungen: Skandalisierung von Arbeitsrechtsver- } \\
\text { letzungen }\end{array}$ \\
\hline
\end{tabular}

Quelle: Eigene Darstellung.

Insgesamt interagierten so vorherige Verhandlungen mit Kampagnen, die vor allem von NGOs des Bündnisses getragen werden, und richteten diese neu aus. Mit einem dynamischen Ansatz kann den verschiedenen Effekten der multiplen Mobilisierungsversuche Rechnung getragen werden (McDonnell, King \& Soule, 2015). Unternehmen reagierten auf Druck von sozialen Bewegungen, indem sie CSR-Maßnahmen in ihrem Unternehmen integrierten. Diese Maßnahmen wiederum, wie im Falle von EUROBAN deutlich zu sehen ist, „,increase a firm's receptivity to future activist challenges“ (McDonnell, King \& Soule 2015, S. 654). Dies bedeutet, je mehr die adressierten Unternehmen sich dem Dialog öffneten, umso effektiver schienen die Bemühungen des Bündnisses zu sein. Verhandlungserfolge sollten bei EUROBAN nun nicht durch weitere konfrontative Kampagnen gefährdet werden. Der folgende Adressatenwandel hatte somit nicht nur mit einer gestiegenen Macht der Supermärkte in den Industrien zu tun, sondern ging auf Grundlage etablierter Dialogstrategien vonstatten.

Abschließend lässt sich somit festhalten, dass in dem Gewerkschafts-NGO-Bündnis Strategien auf spezifische Weise interagierten. Vorherige Strategien und deren Ergebnisse 
erwiesen sich hier als neue strategische Ansatzpunkte. Durch den Interaktionsmechanismus Verhandlungen durch Kampagnen wirkten Strategien des transnationalen Aktivismus auf Strategien des transnationalen sozialen beziehungsweise tripartistischen Dialogs ein. Vice versa bildeten letztere die Ausgangsbasis für erstere durch den Mechanismus Verhandlungen statt Kampagnen. Weitere Interaktionsmechanismen des EUROBAN-Bündnisses (Kryst, 2017) sind zum einen Lobbyingstrategien, bei dem das Bündnis auf marktkonstituierenden Standards aufbaute, um Forderungen an staatliche Entscheidungsträger zu stellen. Zum anderen konnte die zunehmende Einbindung von staatlichen Akteuren in vorherige private Governancebemühungen gezeigt werden, wie sie auch bei anderen Beispielen zu sehen ist (Kryst, 2012; Fransen \& Burgoon, 2015; Kryst \& Zajak, 2017). Daneben war EUROBAN am Aufbau privater Regulierungsinitiativen beziehungsweise Multi-Stakeholder-Initiativen (Mena \& Waeger, 2014, S. 1092) beteiligt und nutzte diese als neue Gelegenheiten für nachfolgende Outsider-Aktionen im Sinne von ,,accountability politics“ (Keck \& Sikkink, 1998).

Diese Ergebnisse stärken das Argument von verschränkten Strategien der Institutionenbildung auf transnationaler Ebene. Strategische Interaktionen sind sowohl im Zeitverlauf zu erkennen als auch parallel in so genannten „trajectories of mobilization“ (Alfinito Vieira \& Quack, 2016) oder „transnational pathways“ (Zajak, 2017), die sich gegenseitig beeinflussen: ,actors situated in one trajectory picked up on the outcomes of other trajectories in order to forward their strategies and projects“ (Alfinito Vieira \& Quack 2016, S. 392). Die hier beschriebenen Mechanismen zeigen, auf welche Weise in GewerkschaftsNGO-Bündnissen vorherige Strategien zu Ausgangspunkten für neue Strategien werden.

Gewerkschaften und NGOs bringen dabei ihre spezifischen Funktionen ein. Die Unterscheidung zwischen strategischen Präferenzen von Gewerkschaften und NGOs stimmt mit jüngsten Beobachtungen einer unterschiedlichen Strategiebasis überein. Gewerkschaftliches Handeln wird auf der Basis von Arbeitnehmermobilisierung charakterisiert, während NGOs die Mobilisierung von Konsumenten für ihre Aktionen favorisieren (Egels-Zandén, Lindberg \& Hyllman, 2015, S. 350f.). Diese Beobachtung endet jedoch nicht notwendigerweise im Konflikt. Zum Beispiel können Gewerkschaften - zumindest für eine gewisse Zeit damit einverstanden sein, gemäß einer NGO-Strategie zu agieren oder eine passive Rolle in Bündnissen einzunehmen (Egels-Zandén, Lindberg \& Hyllman 2015, S. 354). Das hier aufgeführte Beispiel zeigt darüber hinaus, wie Strategieinteraktionen zu zeitlich parallelen oder kausal bedingten Kombinationen der beiden Strategietypen führen. Strategien von Gewerkschaften und NGOs interagieren gewinnbringend in Bündnissen.

\section{Fazit}

Innerhalb von Gewerkschafts-NGO-Bündnissen interagieren Strategien in der globalen Governancearchitektur von Arbeit. Aufbauend auf einem Analyserahmen von Aktionen, Adressaten und Forderungen, arbeite ich auf der einen Seite Strategien des transnationalen sozialen beziehungsweise tripartistischen Dialog heraus, die auf Insider-Aktionen bauen und staatliche Adressaten sowie Unternehmen in den industriellen Beziehungen mit spezifischen Forderungen konfrontierten. Auf der anderen Seite werden Strategien des transnationalen Aktivismus benannt, die Outsider-Aktionen verwenden, um über die Öffentlichkeit 
oder Marktakteure weitreichende Forderungen für private Governance von Arbeit zu stellen. Gewerkschaften bevorzugen ursprünglich erstere Strategien, während NGOs letztere favorisieren. Doch trotz der spezifischen Präferenzen, die die Akteursgruppen einbringen, sind Graubereiche und gegenseitige Unterstützungen sichtbar. Durch die Darstellung eines langanhaltenden Bündnisses kann gezeigt werden, wie über die Zeit Strategien auf transnationaler Ebene kombiniert werden. Ich identifiziere dabei beispielhaft zwei Interaktionsmechanismen, die strategische Entwicklungen im Zeitverlauf abbilden: Verhandlungen durch Kampagnen und Verhandlungen statt Kampagnen.

Mit diesen Ergebnissen trage ich zur Forschung von Gewerkschafts-NGO-Bündnissen bei. Während Kooperationen von Gewerkschaften und NGOs eine gegenseitige Unterstützung nahelegen, konzentrieren sich bisherige Arbeiten meist auf die Phase der Bündnisbildung. Nur wenige Ausnahmen untersuchen gemeinsame Strategieentwicklungen - seien es konfliktreiche (Ford, 2009; Egels-Zandén, Lindberg \& Hyllman, 2015) oder erfolgreiche (Kryst, 2012). Meine Ergebnisse verweisen auf distinktive Strategien von Gewerkschaften und NGOs, wobei jedoch Überlappungen und vor allem interagierende Strategien empirisch evident sind. Dies bedeutet, dass Gewerkschaften und NGOs an ihren originär strategischen Präferenzen festhalten können und sich nicht zwingend auf eine gemeinsame Strategie einigen müssen, sondern - im Gegenteil - ihre unterschiedlichen Strategien sich kausal bedingen. Im vorliegenden Beitrag wurde ein langanhaltendes Bündnis auf seine Strategieinteraktionen hin untersucht. Inwiefern gegenteilige Mechanismen bei nicht komplementären Strategien vorhanden sind, bedarf der Untersuchung weiterer Fallbeispiele. Ähnliche Mechanismen, wie die hier identifizierten, könnten zudem bei reinen NGO- oder reinen Gewerkschaftsbündnissen auftreten. Weitere Forschung müsste hier die Strategiebedingungen, die Gewerkschaften und NGOs mit spezifischen Funktionen in Bündnissen ausstatten, weiter konkretisieren.

Daneben verweist dieser Beitrag auf Forschung zu öffentlicher und privater Governance in der Arbeitsregulierung. Bei der Übersetzung des interaktionistischen Strategiebegriffs in das Feld der Arbeitsregulierung ist es notwendig, neue Formen der privaten Governance in das Handlungsspektrum von Gewerkschaften und NGOs zu integrieren. Dies ist hier in der Form von markt-konstituierenden Aktionsformen als eine Outsider-Aktion geschehen, sowie in der Benennung der Marktsphäre als neue politische Arena. Eine klare Abgrenzung von staatlicher gegenüber privater Governancestrategie wird des Weiteren als zu überspitzt abgelehnt, da es zu kombinierten Formen von öffentlicher und privater Governance durch nichtstaatliche Akteure kommt (Fransen \& Burgoon, 2015; Kryst \& Zajak, 2017).

Zuletzt trägt diese Studie zur Weiterentwicklung eines strategisch-interaktionistischen Ansatzes nicht nur in der Governancearchitektur von Arbeit bei (Jasper, 2004; den Hond, Stolwijk \& Merk, 2014). Strategien sind im politischen Raum omnipräsent. Ob es um Kampagnen von NGOs oder um Verhandlungen von Gewerkschaften mit Arbeitgeberverbänden geht: Die politischen Akteure stellen mittels ausgewählter Aktionen ihre Forderungen an bestimmte Adressaten, um deren Handeln zukünftig zu beeinflussen. Oder anders: Sie versuchen ihr Gegenüber zum gewünschten Handeln zu bewegen. Sie agieren strategisch. Jüngste Forschung betont die Notwendigkeit, dynamische Interaktionen mit in Betracht zu ziehen, um ein umfassenderes Bild von Arbeitsaktivisten in der globalen Arbeitsregulierung zu geben (Den Hond, de Bakker \& de Haan 2010, S. 653; McDonnell, King \& 
Soule 2015, S. 672). Ein interaktiver Strategieansatz analysiert kausale und dynamische Zusammenhänge der Strategieentwicklung und zielte hier auf eine Beschreibung der Interaktionsmechanismen zwischen Strategien von Gewerkschaften und NGOs ab.

\section{Literatur}

Abendroth, W. (1969). Sozialgeschichte der europäischen Arbeiterbewegung. 5. Aufl. Frankfurt/Main: Suhrkamp.

Alfinito Vieira, A. C., \& Quack, S. (2016). Trajectories of Transnational Mobilization for Indigenous Rights in Brazil. Revista de Administração de Empresas, 46 (4), 380-394. doi: 10.1590/S0034-759020160403

Anner, M. \& Evans, P. (2004). Building Bridges across a Double Divide: Alliances between US and Latin American Labour and NGOs. Development in Practice, 14, 34-47. doi: 10.1080/0961452032000170613

Arenas, D., Lozano, J. M. \& Albreda, L. (2009). The Role of NGOs in CSR: Mutual Perceptions among Stakeholders. Journal of Business Ethics, 88, 175-197. doi: 10.1007/s10551-009-0109-x

Avdagić, S. \& Crouch, C. (2006). Organized Economic Interests: Diversity and Change in an Enlarged Europe. In P. Heywood, E. Jones, M. Rhodes \& U. Sedelmeier (Hrsg.), Developments in European Politics (S. 196-215). Houndmills: Palgrave Macmillan.

Baccaro, L., Hamann, K. \& Turner, L. (2003). The Politics of Labour Movement Revitalization: The Need for a Revitalized Perspective. European Journal of Industrial Relations 9 (1), 119-133. doi: $10.1177 / 0959680103009001455$

Balsiger, P. (2015). Managing Protest: The Political Action Repertoires of Corporations. In D. Della Porta \& M. Diani (Hrsg.), The Oxford Handbook of Social Movements (S. 653-665). Oxford: Oxford University Press.

Bartley, T. (2007). Institutional Emergence in an Era of Globalization: The Rise of Transnational Private Regulation of Labour and Environmental Conditions. American Journal of Sociology, 113 (2), 297-351. doi: 10.1086/518871

Bergmann, J., Jacobi, O. \& Müller-Jentsch, W. (1975). Gewerkschaften in der Bundesrepublik. Gewerkschaftliche Lohnpolitik zwischen Mitgliederinteressen und ökonomischen Sachzwängen. Frankfurt: Europäische Verlagsanstalt.

Braun, R. \& Gearhart, J. (2004). Who Should Code your Conduct? Trade Union and NGO Differences in the Fight for Workers' Rights. Development in Practice, 14, 183-196. doi: 10.1080/0961452032000170758

Bronfenbrenner, K. (Hrsg.) (2007). Global Unions: Challenging Transnational Capital Through Cross-Border-Campaigns. Ithaca: Cornell University Press.

Brookes, M. (2013). Varieties of Power in Transnational Labor Alliances. An Analysis of Workers' Structural, Institutional, and Coalitional Power in the Global Economy. Labor Studies Journal, 38 (3), 181-200. doi: 10.1177/0160449X13500147

Clemens, E. (2005). Two Kinds of Stuff. The Current Encounter of Social Movements and Organisations. In G. F. Davis (Hrsg.), Social Movements and Organization Theory (S. 351-366). Cambridge: Cambridge University Press. doi: 10.1017/CBO9780511791000.018

Cohen, L. \& Rai, S. (2000). Global Social Movements. Towards a Cosmopolitan Politics. In R. Cohen, \& S. Rai (Hrsg.), Global Social Movements, (S. 1-17). London: Athlone Press.

Compa, L. (2004). Trade unions, NGOs, and corporate codes of conduct. Development in Practice, 14 (1/2), 210-215. doi: 10.1080/0961452032000170776

Della Porta, D. (2006). Form Corporatist Unions to Protest Unions? On the (difficult) Relations between Organized Labour and new Social Movements. In C. Crouch \& W. Streeck (Hrsg.), The 
Diversity of Democracy. Corporatism, Social Order and Political Conflict (S. 71-97). Cheltenham: Edward Elgar.

Den Hond, F. \& de Bakker, F. (2012): Boomerang politics: how transnational stakeholders impact multinational corporations in the context of globalization. In A. Lindgreen, P. Kotler, J. Vanhamme \& F. Maon (Hrsg.), A Stakeholder Approach to Corporate Social Responsibility: Pressures, Conflicts, Reconciliation (S. 275-292). Aldershot: Gower.

Den Hond, F., de Bakker, D. \& de Haan, P. (2010). The Sequential Patterning of Tactics. Activism in the Global Sports Apparel Industry, 1988-2002. International Journal of Sociology and Social Policy, 30 (11/12), 648-665. doi: 10.1108/01443331011085240

Den Hond, F., Stolwijk, S. \& Merk, J. (2014). A strategic-interaction analysis of an Urgent Appeal System and its outcomes for garment workers. Mobilization: An International Quaterly, 19 (1), 83-111.

Eisinger, P. K. (1973). The Conditions of Protest Behavior in American Cities. American Political Science Review, 67 (1), 11-28. doi: 10.2307/1958525

Egels-Zandén, N. \& Hyllman, P. (2011). Differences in Organizing between Unions and NGOs: Conflict and Cooperation among Swedish Unions and NGOs. Journal of Business Ethics, 101, 249261. doi: 10.1007/s10551-010-0720-x

Egels-Zandén, N., Lindberg, K. \& Hyllman, P. (2015). Multiple Institutional Logics in Union-NGO Relations: Private Labor Regulation in the Swedish Clean Clothes Campaign. Business Ethics: A European Review, 24 (4), 347-360. doi: 10.1111/beer.12091

Ehmke, E., Simon, A. \& Simon, J. (2009). Internationale Arbeitsstandards im globalen Kapitalismus. In E. Ehmke, A. Simon \& J. Simon (Hrsg.), Internationale Arbeitsstandards in einer globalisierten Welt (S. 12-42). Wiesbaden: VS-Verlag für Sozialwissenschaften.

EUROBAN (2003). Minutes of EUROBAN Meeting, Part II. Montreuil/France, 11.-12. Dezember 2003.

Evans, P. (2010). Is it Labor's Turn to Globalize? Twenty-first Century Opportunities and Strategic Responses. Global Labour Journal, 20, 352-379. doi: 10.15173/glj.v1i3.1082

Fairbrother, P. \& Webster, E. (2008). Social Movement Unionism: Questions and Possibilities. Employee Responsibilities and Rights Journal, 20 (4), 309-313. doi: 10.1007/s10672-008-9091-1

Fichter, M., Gester, J. \& Zeuner, B. (2004). Zukunft der Gewerkschaften. Externe Herausforderungen - Interne Problemlagen - Zukunftsoptionen: Eine internationale Perspektive. Arbeitspapier Hans-Böckler-Stiftung, 44 (Teil II), 113-186.

Ford, M. (2009). Workers and Intellectuals. NGOs, Trade Unions and the Indonesian Labour Movement. Singapore: NUS Press.

Fransen, L. \& Burgoon, B. (2015). Global Labour-Standards Advocacy by European Civil Society Organizations: Trends and Developments. British Journal of Industrial Relations, 53, 204-230. doi: 10.1111/bjir.12017

Frege, C. M. \& Kelly, J. (2003). Union Revitalization Strategies in Comparative Perspective. European Journal of Industrial Relations, 9, 7-24. doi: 10.1177/095968010391002

Frundt, Henry J. (2009). Fair Bananas. Famers, Workers, and Consumers Strive to Change an Industry. Tucson, AZ: University of Arizona Press.

Gallin, D. (2000). Trade Unions and NGOs: A Necessary Partnership for Social Development. United Nations Research Institute for Social Development: Civil Society and Social Movement Program, 1.

George, A. L. \& Bennett, A. (2005). Case Studies and Theory Development in the Social Sciences. Cambridge: MIT Press.

Glaab, M. (2008). Analyse: ,Leadership matters` - auch und gerade in Strategiefragen. Forschungsjournal Neue Soziale Bewegungen, 21 (1), 97-101. 
Hassel, A. (2008). The Evolution of a Global Labor Governance Regime. Governance: An International Journal of Policy, Administration, and Institutions, 21 (2), 231-251.

doi: 10.1111/j.1468-0491.2008.00397.x

Heins, V. (2002). Weltbürger und Lokalpatrioten: Eine Einführung in das Thema Nichtregierungsorganisationen. Opladen: Leske + Budrich. doi: 10.1007/978-3-663-11827-5

Janett, D. (1997). Vielfalt als Strategievorteil: Zur Handlungskompetenz von Nicht-Regierungsorganisationen in komplexen Umwelten. In E. Altvater, A. Brunnengräber, M. Haake \& H. Walk (Hrsg.), Vernetzt und verstrickt. Nicht-Regierungs-Organisationen als gesellschaftliche Produktivkraft (S. 146-173). Münster: Westfälisches Dampfboot.

Jasper, J. M. (2004). A Strategic Approach to Collective Action: Looking for Agency in Social Movement Choices. Mobilization, 9, 1-13.

Jasper, J. M. (2012). Choice Points, Emotional Batteries, and Other Ways to Find Strategic Agency at the Microlevel. In G. M. Maney, R. V. Kutz-Flamenbaum, D. A. Rohlinger \& J. Godwin (Hrsg.), Strategies for Social Change (S. 23-42). Minneapolis, London: University of Minnesota Press. https://doi.org/10.5749/minnesota/9780816672899.003.0002

Jasper, J. M. \& Duyvendak, J. W. (Hrsg.) (2015). Players and Arenas: The Interactive Dynamics of Protest. Amsterdam: Amsterdam University Press.

Keck, M. E. \& Sikkink, K. (1998). Activists beyond Boarders: Advocacy Networks in International Politics. Ithaca, New York: Cornell University Press.

King, B. G. (2008). A Social Movement Perspective of the Stakeholder Collective Action and Influence. Business and Society, 47, 21-49. doi: 10.1177/0007650307306636

King, B. G. \& Pearce, N. A. (2010). The Contentiousness of Markets: Politics, Social Movements, and Institutional Change in Markets. Annual Review of Sociology, 35 (1), 249-267. doi: 10.1146/annurev.soc.012809.102606

Klein, A., Walk, H. \& Brunnengräber, A. (2005). Mobile Herausforderer und alternative Eliten. NGOs als Hoffnungsträger einer demokratischen Globalisierung? In A. Brunnengräber, A. Klein \& H. Walk (Hrsg.), NGOs im Prozess der Globalisierung. Mächtige Zwerge - umstrittene Riesen (S. 10-77). Wiesbaden: VS-Verlag. doi: 10.1007/978-3-322-80983-4_2

Koch-Baumgarten, S. (1999). Gewerkschaftsinternationalismus und die Herausforderung der Globalisierung. Das Beispiel der Internationalen Transportarbeiterföderation (ITF). Frankfurt/Main: Campus Verlag.

Koch-Baumgarten, S. (2006). Globale Gewerkschaften und Industrielle Beziehungen in der Global Governance. Industrielle Beziehungen, 13 (3), 205-222.

Koch-Baumgarten, S. (2011). Gewerkschaften und global governance: Grenzen und Möglichkeiten einer grenzüberschreitenden Regulierung von Erwerbsarbeit. Internationale Politik und Gesellschaft, 2, 51-68.

Koch-Baumgarten, S., \& Kryst, M. (2015). Trade unions and Collective Bargaining Power in Global Governance. In A. Marx, J. Wouters, G. Rayp \& L. Beke (Hrsg.), Global Governance of Labour Rights. Assessing the Effectiveness of Transnational Public and Private Policy Initiatives (S. 150-169). Cheltenham, Northhampton: Edward Elgar. doi: 10.4337/9781784711467.00013

Krüger, S. (2002). Nachhaltigkeit als Kooperationsimpuls. Sozial-ökologische Bündnisse zwischen NGOs und Gewerkschaften. Münster: Westfälisches Dampfboot.

Kryst, M. (2012). Coalitions of Labor Unions and NGOs: The Room of Maneuver of the German Clean Clothes Campaign. Interface: A Journal for and about Social Movements, 4 (2), 101-129.

Kryst, M. (2017). Transnationale Bündnisse von Gewerkschaften und NGOs: Eine interaktionistische Strategieanalyse. Dissertation Philipps-Universität Marburg, unveröffentlicht.

Kryst, M. \& Zajak, S. (2017). Mehr Staat durch Markt? Adressierungsstrategien der Anti-SweatshopBewegung in Europa. In P. Daphi, N. Deitelhoff, D. Rucht, D. \& S. Teune (Hrsg.): Protest in 
Bewegung? Zum Wandel von Bedingungen, Formen und Effekten politischen Protests. Sonderband: Leviathan. Baden-Baden: Nomos, S. 63-92.

doi: 10.5771/9783845288413-62

Maney, G. M., Andrews, K. T., Kutz-Flamenbaum, R. V., Rohlinger, D. A., \& Goodwin, J. (2012). An Introduction to Strategies for Social Change. In G. M. Maney, R. V. Kutz-Flamenbaum, D. A. Rohlinger \& J. Goodwin (Eds.), Strategies for Social Change (pp. xi-xxxviii). Minnesota: University of Minnesota Press. doi: 10.5749/minnesota/9780816672899.001.0001

Mayer, B. (2009). Cross-Movement Coalition Formation: Bridging the Labor-Environment Divide. Sociological Inquiry, 79 (2), 219-239. doi: 10.1111/j.1475-682X.2009.00286.x

Mayntz, R. (2004). Mechanisms in the Analysis of Social Macro-Phenomena. Philosophy of the Social Sciences, 34 (2), 237-259. doi: 10.1177/0048393103262552

McDonnell, M.-H., King, B. G. \& Soule, S. (2015). A Dynamic Process Model of Private Politics: Activists Targeting and Corporate Receptivity to Social Challenges. American Sociological Review, 80 (3), 648-678. doi: 10.1177/0003122415581335

Mena, S. \& Waeger, D. (2014). Activism for Corporate Responsibility: Conceptualizing Private Regulation Opportunity Structures. Journal of Management Studies, 36, 249-267. doi: doi.org/10.1111/joms.12092

Meyer, D. S. \& Staggenborg, S. (2012). Thinking about Strategy. In G. M. Maney, R. V. Kutz-Flamenbaum, D. A. Rohlinger \& J. Goodwin (Hrsg.), Strategies for Social Change (S. 3-22). Minnesota: University of Minnesota Press. doi: 10.5749/minnesota/9780816672899.003.0001

Müller, T., Platzer, H.-W. \& Rüb, S. (2010). Transnational company policy and coordination of collective bargaining - new challenges and roles for European industry federations. In: Transfer, 16 (4), 509-524. doi: 10.1177/1024258910383083

Müller-Jentsch, W. (2008). Arbeit und Bürgerstatus. Studien zur sozialen und industriellen Demokratie. Wiesbaden: VS-Verlag.

Münkler, H. (2010). Zum Verhältnis von politischer und militärischer Strategie. In J. Raschke \& R. Tils (Hrsg.), Strategie in der Politikwissenschaft. Konturen eines neuen Forschungsgebiets (S. 45-73). Wiesbaden: VS Springer. doi: 10.1007/978-3-531-92209-6_3

Parker, L. \& Harrison, J. on behalf of EUROBAN (2004). Alternative Trade Policy Proposal: Differentiated Tariffs.

Pries, L. (2008). Die Transnationalisierung der sozialen Welt. Frankfurt/Main: Suhrkamp.

Pries, L. (2010). Erwerbsregulierung in einer globalisierten Welt. Wiesbaden: VS-Verlag. doi: 10.1007/978-3-531-91956-0

Raschke, J. \& Tils, R. (2013). Politische Strategie. Eine Grundlegung. 2. Aufl. Wiesbaden: VSVerlag. doi: 10.1007/978-3-531-19871-2

Rehder, B. (2008). Revitalisierung der Gewerkschaften? Die Grundlagen amerikanischer Organisierungserfolge und ihre Übertragbarkeit auf deutsche Verhältnisse. Berliner Journal für Soziologie, 18 (3), 432-456. doi: 10.1007/s11609-008-0029-0

Reutter, W. \& Rütters, P. (2003). Internationale und europäische Gewerkschaftsorganisationen: Geschichte, Struktur und Einfluss. In W. Schroeder \& B. Weßels (Hrsg.), Die Gewerkschaften in Politik und Gesellschaft der Bundesrepublik Deutschland (S. 512-542). Wiesbaden: Westdeutscher Verlag.

Riisgaard, L. (2004). The IUF/COLSIBA - CHIQUITA framework agreement: a case study. Multinational Enterprises Programme Working Paper No. 94.

Roth, R. (2005). Transnationale Demokratie. Beiträge, Möglichkeiten und Grenzen von NGOs. In A. Brunnengräber, A. Klein \& H. Walk (Hrsg.), NGOs im Prozess der Globalisierung. Mächtige Zwerge - umstrittene Riesen (S. 80-128). Wiesbaden: VS-Verlag.

doi: 10.1007/978-3-322-80983-4_3 
Sabatier, P. A. (1998). The Advocacy Coalition Framework: Revisions and Relevance for Europe. Journal of European Public Policy, 5, 98-130. doi: 10.1080/13501768880000051

Saretzki, T. (2010). Strategie als Herausforderung für die deliberative Demokratietheorie. In J. Raschke, \& R. Tils (Hrsg.), Strategie in der Politikwissenschaft. Konturen eines neuen Forschungsfeldes (S. 121-150). Wiesbaden: VS-Verlag. doi: 10.1007/978-3-531-92209-6_6

Scherrer, C. (2002). Von den NGOs lernen? Wie Gewerkschaften den Prozess der Globalisierung beeinflussen können. In C. Flavin, B. Young, C. Scherrer \& K. Zwickel (Hrsg.), Global Governance. Gewerkschaften und NGOs - Akteure für Gerechtigkeit und Solidarität (S. 116-128). Hamburg: VSA-Verlag.

Schmitt-Beck, R. (2008). Professionalisierte Kommunikation. Acht Thesen zum Verhältnis von politischer Strategie und Öffentlichkeit. Forschungsjournal Neue Soziale Bewegungen, 21 (1), 71 73.

Schroeder, W. (2009). Gewerkschaften in drei Welten. Ein Überblick. Forschungsjournal Neue Soziale Bewegungen, 4, 13-22. doi: 10.1515/fjsb-2009-0404

Schroeder, W. (2014). Handbuch Gewerkschaften in Deutschland. Wiesbaden: Springer VS. doi: org/10.1007/978-3-531-19496-7

Schurman, R. (2004). Fighting "Frankenfoods": Industry Opportunity Structures and the Efficacy of the Anti-Biotech Movement in Western Europe. Social Problems, 51 (2), 243-268. doi: org/10.1525/sp.2004.51.2.243

Schwarz, C. (2008). Analyse: Strategic Studies als akademische Disziplin. Forschungsjournal Neue Soziale Bewegungen, 21 (1), 101-107.

Smith, A. (2015): The Banana Saga: Towards fair and sustainable product chains. Unpublished.

Spooner, D. (2004). Trade Unions and NGOs: The Need for Cooperation. Development in Practice, 14, 19-33. doi: org/10.1080/0961452032000170604

Streeck, W. (1996). Industrielle Beziehungen in einer institutionalisierten Wirtschaft. In U. Beck (Hrsg.), Politik der Globalisierung (S. 169-202). Frankfurt/Main: Suhrkamp.

Streek, W. \& Rehder, B. (2005). Institutionen im Wandel: Hat die Tarifautonomie eine Zukunft? In H. W. Busch, H. P. Frey, M. Hüther, B. Rehder \& W. Streeck (Hrsg.), Tarifpolitik im Umbruch (S. 49-82). Köln: Deutscher Institutsverlag.

Tilly, C. (1978). From Mobilization to Revolution. Reading, MA: Addison-Wesley.

Van Dyke, N. \& McCammon, H. J. (Hrsg.) (2010). Strategic Alliances. Coalition Building and Social Movements. Minneapolis: University of Minnesota Press.

Wiesendahl, E. (2010). Rationalitätsgrenzen politischer Strategie. In J. Raschke \& R. Tils (Hrsg.), Strategie in der Politikwissenschaft. Konturen eines neuen Forschungsfeldes (S. 21-44). Wiesbaden: VS-Verlag. doi: org/10.1007/978-3-531-92209-6_2

Zajak, S. (2017). Transnational Activism, Global Labor Governance, and China. New York: Palgrave Macmillan. doi: org/10.1057/978-1-349-95022-5

Zimmer, A. \& Speth, R. (2009). Verbändeforschung. In V. Kaina \& A. Römmele (Hrsg.), Politische Soziologie. Ein Studienbuch (S. 267-309). Wiesbaden: Springer VS.

doi: org/10.1007/978-3-531-91422-0_11 


\section{DuEPublico}

\section{Duisburg-Essen Publications online}

UNIVERSITÄT

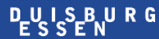

offen im Denken

Ub $\mid \begin{aligned} & \text { universitäts } \\ & \text { bibliothek }\end{aligned}$

Dieser Text wird via DuEPublico, dem Dokumenten- und Publikationsserver der Universität Duisburg-Essen, zur Verfügung gestellt. Die hier veröffentlichte Version der E-Publikation kann von einer eventuell ebenfalls veröffentlichten Verlagsversion abweichen.

DOI: $\quad$ 10.3224/indbez.v25i2.05

URN: urn:nbn:de:hbz:464-20210401-124402-4 